\title{
Design of a flow integrating information system based on cloud computing
}

\author{
Li Ren-jie ${ }^{1}$, Jiang Jian ${ }^{1} \&$ Chen Wei ${ }^{2}$ \\ ${ }^{1}$ Beijing Institute of Technology, Beijing 100081, China \\ ${ }^{2}$ Chinese Academy of Sciences, Beijing 100190, China
}

Keywords: flow integrating, cloud computing, Hadoop, cloud application.

Abstract. Vast amounts of data is collected into flow measurement system for the primary purpose of trade settlement, the fairness and justice of entire trade settlement system depends entirely on the integrity of the flow integrating system. Therefore, the role of flow integrating system is very important. Measurement equipment of traditional flow integrating system is very expensive, but the information release ability is poor and the operation and maintenance of the system is complex. To solve this problem, this paper design a flow integrating information system based on cloud computing technology. The design uses a cloud data centre based on Hadoop to manage massive data, cloud mathematical models to compute flow information and cloud application platform to publish the information to users. Actual operating data of Guangzhou Longxue shipyard shows that calculation error of the system is less than $0.1 \%$ with a very small delay time about 50 Ms when the amount of data is more than 1 million, and users can get information of the system through cloud applications conveniently and quickly.

\section{Introduction}

Gas is one of the most common materials used in industrial production. It requires millions of flow integrating in the whole process of collecting, storage, transportation and disposal of gas. Some of flow integrating involves a huge amount of money in trade settlement[1], so it requires very high measurement accuracy and reliability. In the past, the structure and function of flow system is very simple because of low intelligent degree of instruments, poor Information interaction ability of the equipment, and bad Management of the use of gas. A large number of data is trapped in instruments in the production site[2], separated from each other. Along with the continuous development of industrial modernization, more and more detecting instruments appear in the Gas pipeline network .The amount of data is increasing in a geometrical progression and demand for analysis of the data becomes diversification at the same time[3]. So, flow integrating information system plays a more and more important role in industrial informatization construction. Taking Longxue shipyard as an example, there are about 3000 measuring instruments related to flow detection in production field, which means it would produce nearly one million record data every day. Therefore, higher and higher petitions of reliable access to massive data, high performance computing, stability and scalability of the system and diversification of applications were asked. With the integration of our country's industrial modernization and informatization, it is need to design a new flow integrating information system.

This paper uses cloud computing to design a new flow integrating information system on the basis of the instruments which already exist in the production site. When in use, the design of the flow integrating information system shows many advantages over the old system. Firstly, the design uses cloud storage technology to store, retrieve and manage massive data, which would enhance speed and reliability of the system. Secondly, the system get Analysis of flow data through cloud mathematical models accurately and efficiently. Finally, the design uses cloud application platform which would guarantee the stability and expansibility of the system. 


\section{Design of the system}

\section{The overall system architecture}

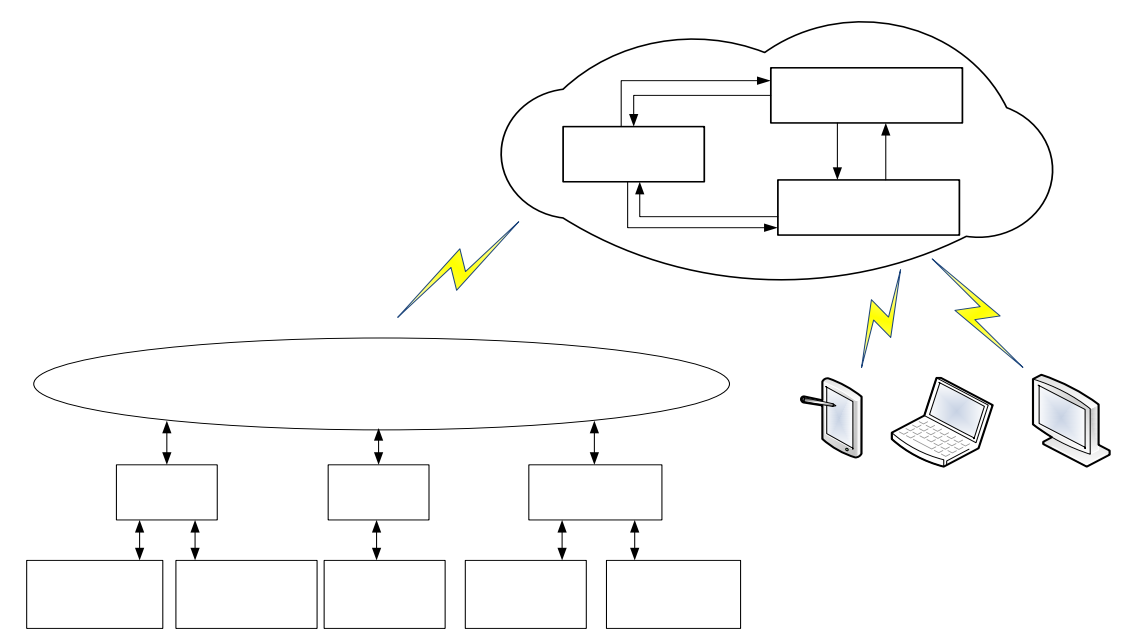

Fig.1: The overall architecture of the flow integrating information system

The design takes the status of instruments and the actual environmental conditions as design basis. The flow integrating information system gets flow, pressure, temperature etc. data from production field, and then exploits cloud computing technology to analyse and manage the data. Finally, the system offers users information services through cloud applications. The overall system architecture is shown in Fig.1.

The cloud centre set up in high performance server, using IAAS[4] (Infrastructure as a Service) cloud service model. Cloud centre consists of a cloud data centre, cloud mathematical models and cloud applications. The cloud data centre is responsible to manage data from production field, analysis from mathematical models and log generated by cloud applications. Cloud mathematical models calculate flow information accurately with basic data from cloud data centre. Meanwhile, cloud application platform provide real-time flow information through web browsers, PC clients and mobile applications.

\section{Cloud data centre}

Cloud data center is responsible to manage all of the data of the system. The design of cloud data center adopts Hadoop technology framework, using HBase as a data management system, HDFS[5](Hadoop distributed file system) as a distributed storage system, Xen as the virtual machine. Cloud data center uses the Master/Slave architecture to construct the cluster[6], which consists of a master server and a number of data servers. Architecture of cloud data center is shown in Fig.2:

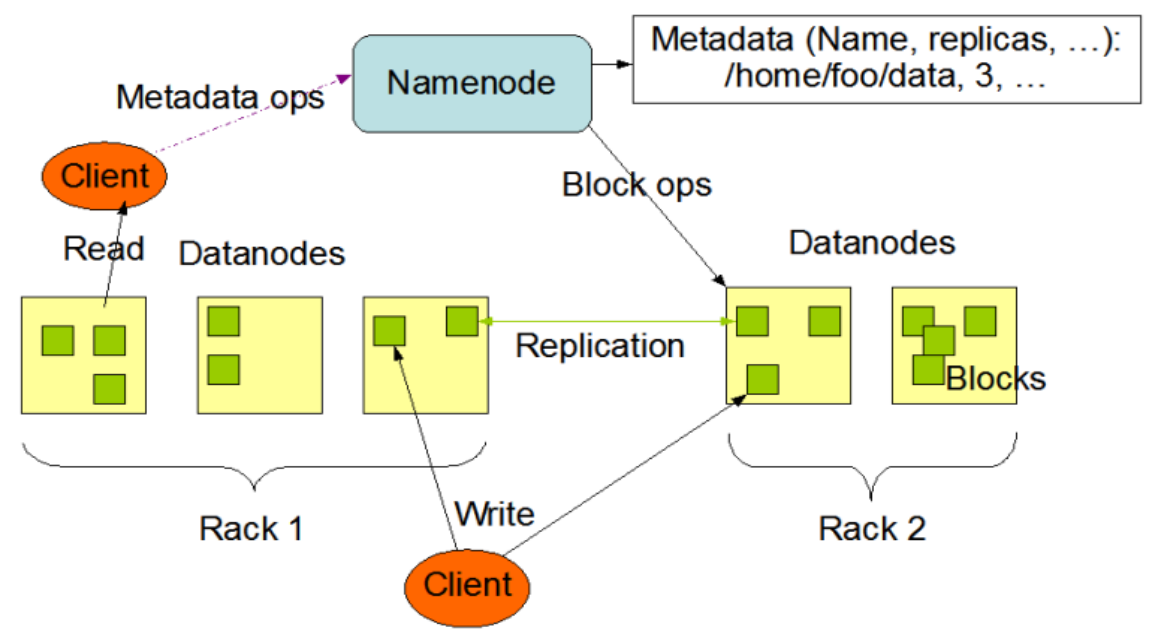

Fig.2: The architecture of cloud data centre 
Namenode stands for the master server, which is used to manage file system metadata. Namenode executes the operations associated with namespace of file system, such as Opening, closing, renaming the file or directory. Datanode stands for data server, which stores the actual data. Datanode is responsible for dealing with read and write requests of the clients and executing create, copy, delete act operations of data block according to the command from Namenode[7].

\section{Cloud mathematical models}

After obtaining the flow, pressure, temperature etc. data of locale instruments, the system uses corresponding mathematical models to calculate the instantaneous flow and integral base on temperature and pressure compensation principle. According to the output types of flow meters, flow signal can be divided into the differential pressure flow signal, linear flow signal and pulse flow signal[8].

The basic formulas of differential pressure flow signal are:

$$
\begin{aligned}
& Q_{m}=k \sqrt{\Delta p \cdot \rho} \\
& Q_{v}=k \sqrt{\Delta p \cdot \rho}
\end{aligned}
$$

In the formula, $\mathrm{Qm}$ is the mass flow value of the corresponding differential pressure flow signal; $\mathrm{k}$ is the flow coefficient of flow meter; $\Delta P$ is the output value of differential pressure flow meters; $\rho$ is the density of the gas; $\mathrm{Qv}$ is the volume flow value of the corresponding differential pressure flow signal. The output of linear signal flow meter has a linear relation with the gas flow, and we can get the following formula[9]:

$$
Q=G
$$

In the formula, $Q$ is the flow value after integrating; $G$ is the measured value of linear signal flowmeter. The basic formula of pulse flow signal is given as follow:

$$
Q=\frac{3600 f}{k}
$$

In the formula, $\mathrm{f}$ is the pulse frequency of flow signal.

The major mathematical models of flow integrating compensation could be classified into two broad categories: general gas or steam.

\section{Mathematical models of general gas}

If the output of flow meter is differential pressure signal, we can deduce that temperature and pressure compensation equation for differential pressure signal by ideal gas equation[10]:

$$
Q_{n}=Q_{\max } \cdot \sqrt{\frac{\Delta P}{\Delta P_{\max }} \cdot \frac{\left(P+P_{A}\right)\left(T_{s}+T_{0^{\circ} \mathrm{C}}\right)}{\left(P_{s}+P_{A}\right)\left(T+T_{0^{\circ} \mathrm{C}}\right)}}
$$

Similarly, we can get the formula of linear signal. When linear signal representing the volume flow in the actual working conditions, we can get formula (6):

$$
Q_{n}=Q_{f} \cdot \frac{\left(P+P_{A}\right)\left(T_{n}+T_{0^{\circ} \mathrm{C}}\right)}{P_{n}\left(T+T_{0^{\circ} \mathrm{C}}\right)}
$$

When linear signal representing the volume flow after fixed-point compensation, we can get formula (7):

$$
Q_{n}=Q_{s} \cdot \frac{\left(P+P_{A}\right)\left(T_{s}+T_{0^{\circ} \mathrm{C}}\right)}{\left(P_{s}+P_{n}\right)\left(T+T_{0^{\circ} \mathrm{C}}\right)}
$$

If the output of flow meter is pulse flow signal, the flow value get from flow meter has nothing to do with density of gas and is usually volume flow in the actual working conditions. So we can get volume flow in standard conditions through the temperature and pressure compensation. The formula of general gas is given as follow:

$$
Q_{n}=\frac{3600 f}{k} \cdot \frac{\left(P+P_{A}\right)\left(T_{n}+T_{0^{\circ} \mathrm{C}}\right)}{P_{n}\left(T+T_{0^{\circ} \mathrm{C}}\right)}
$$


In the above formulas, Qn is the volume flow in standard conditions; Qs is the volume flow after fixed-point compensation in design conditions; Qf the volume flow in working conditions; Pn is the normal atmosphere; Ps the manometer pressure; PA is the local average atmospheric pressure; $\mathrm{T}$ is the temperature in actual working conditions; Tn is the temperature in standard conditions; Ts is the temperature in design conditions; $\mathrm{T} 0^{\circ} \mathrm{C}$ is the absolute temperature of $0{ }^{\circ} \mathrm{C}$; $\left({ }^{*}\right)$ max is the maximum value of one parameter. Formula (5) (8) constitute temperature and pressure compensation mathematical models of general gas together.

\section{Mathematical models of steam}

Steam is measured by mass flow and doesn't conform to the ideal gas state equation, which is quite different from general gas.

In general, instruments get the volume flow in actual working conditions. The design uses lookup table method to get steam density. Finally, we can calculate mass flow by the volume flow and density. If the output of flow meter is differential pressure signal, we can get formula (9):

$$
Q_{m}=Q_{\max } \cdot \sqrt{\frac{\Delta P}{\Delta P_{\max }} \cdot \frac{\rho}{\rho_{s}}}
$$

If the output of flow meter is linear signal. We can get formula (10) when linear signal representing the volume flow in the actual working conditions:

$$
Q_{m}=Q_{f} \cdot \rho
$$

When linear signal representing the volume flow after fixed-point compensation, we can get formula (11):

$$
Q_{m}=Q_{s} \cdot \frac{\rho}{\rho_{s}}
$$

If the output of flow meter is pulse flow signal, we can get formula (12):

$$
Q_{m}=\frac{3600 f}{k} \cdot \rho
$$

Formula (9) (12) constitute temperature and pressure compensation mathematical models of steam together.

\section{Cloud application platform}

Cloud application platform integrates related resources, which greatly enhance the scalability and the stability of the system. The design separated flow information application platform into service layer and application layer based on information security architecture. Service layer provide business data for application layer. Meanwhile, application layer could display information in various ways, such as web browsers, PC clients, or apps on handsets and tablets.

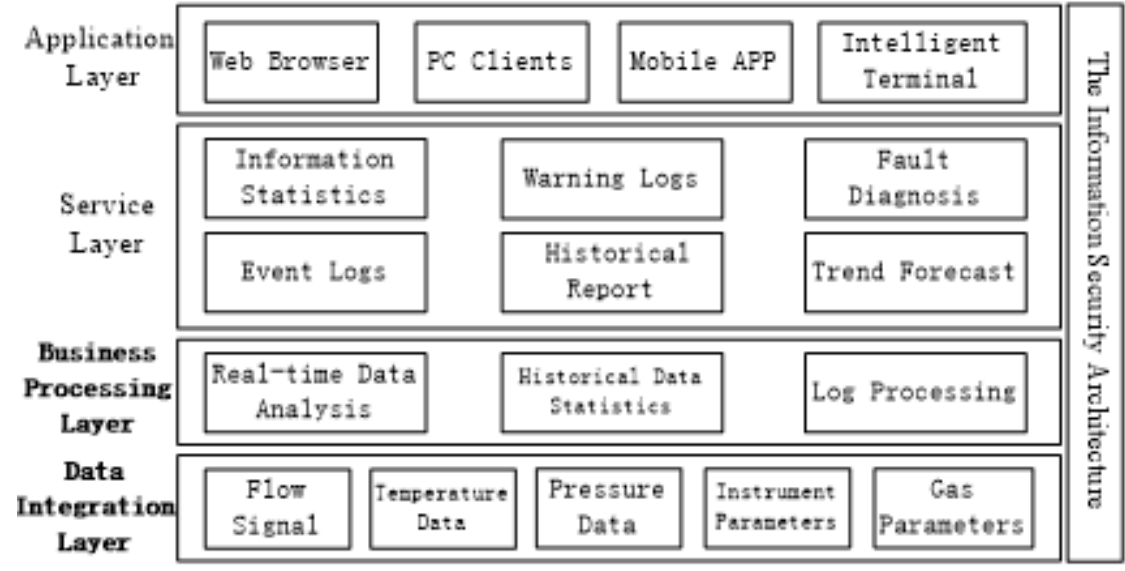

Fig.3: The architecture of cloud data centre

Cloud application platform can achieve open effect. Data interaction of the old flow information system relies on man-made style, which makes the low utilization rate of data and poor information 
interaction. The design of the flow integrating information system based on cloud computing uses cloud application platform to provide flow information service to authorized users in any time and place.

Cloud application platform is with high reliability and maintainability. The traditional flow information system costs huge investment and is difficult to maintain. After using cloud application platform, the new user only need to hire the corresponding applications with your basic data, such as temperature, pressure, flow and Instrument parameters. All of the calculation and analysis work will completed by the cloud application platform and it can generate reports, analyze trends and write log file automatically. It improves accessibility and stability of the flow integrating information system greatly and lowers the entry barrier for newcomers.

\section{Analysis of the results}

\section{Interpolation function}

In order to test whether the flow integrating information system based on cloud computing can accurately calculate flow information, we use the data get from a high precision flow totalizer in the production field as a contrast. Data used in this paper comes from energy control project in Guangzhou Longxue Shipyard. The relevant parameters are as follows : orifice diameter is $6.25 \mathrm{~mm}$; acutance is 1.0 ; roughness is 1.0 ; settlement temperature is $20^{\circ} \mathrm{C}$; Settlement pressure is $101.325 \mathrm{kPa}$. In terms of the system calculation accuracy, error within $0.1 \%$ is acceptable. The results are shown in table 1 :

Table 1 comparison between flow totalizer and the system

\begin{tabular}{|c|c|c|c|c|c|}
\hline $\begin{array}{c}\text { pressure } \\
\text { differential } \\
(\mathrm{kPa})\end{array}$ & $\begin{array}{l}\text { pressure } \\
(\mathrm{kPa})\end{array}$ & temperature $\left({ }^{\circ} \mathrm{C}\right)$ & $\begin{array}{c}\text { flow } \\
\text { totalizer }\end{array}$ & $\begin{array}{l}\text { mathematical } \\
\text { model }\end{array}$ & error \\
\hline 1 & & -20 & 3.63 & 3.6322 & $0.06 \%$ \\
\hline 5 & 100 & 20 & 7.42 & 7.4183 & $0.02 \%$ \\
\hline 10 & & 60 & 9.70 & 9.6997 & $0.03 \%$ \\
\hline 3 & & -20 & 14.08 & 14.0880 & $0.06 \%$ \\
\hline 10 & 500 & 60 & 22.16 & 22.1642 & $0.02 \%$ \\
\hline 30 & & 20 & 40.44 & 40.4362 & $0.01 \%$ \\
\hline 5 & & 20 & 23.89 & 23.8862 & $0.02 \%$ \\
\hline 50 & 1000 & -20 & 80.88 & 80.9033 & $0.02 \%$ \\
\hline 100 & & 60 & 97.01 & 97.0031 & $0.01 \%$ \\
\hline 10 & & 20 & 79.64 & 79.6393 & $0.0009 \%$ \\
\hline 100 & 5000 & 60 & 228.87 & 228.8905 & $0.01 \%$ \\
\hline 500 & & -20 & 628.83 & 628.8274 & $0.0004 \%$ \\
\hline 50 & & 60 & 237.64 & 237.6553 & $0.01 \%$ \\
\hline 500 & 10000 & -20 & 1035.99 & 1035.9952 & $0.0005 \%$ \\
\hline 1000 & & 20 & 1176.18 & 1176.2555 & $0.006 \%$ \\
\hline
\end{tabular}

It indicates that the calculation error of the flow integrating information system is within $0.1 \%$ in working conditions. What's more, data processing capacity of the system based on cloud computing is far more powerful than the old system. Tests show that it costs 5404.155 seconds to input one million flow information data into the system database, which is about 86.95GB. Data-handling capacity of the system can reach about $189.83 \mathrm{ops} / \mathrm{s}$ and the average delay time of one single operation is about $51.3 \mathrm{~ms}$. Meanwhile, the system based on cloud computing shows incomparable advantages in information display, statistics analysis and generating statements. These advantages are brought by powerful calculating ability, reliable processing performance and flexible expansibility of cloud computing.

\section{Conclusions}


This paper presents the flow integrating information system based on cloud computing. And then show the comparative analysis with a high precision flow totalizer in the production field. The flow integrating information system has been put into trial operation in Guangzhou Longxue Shipyard. The result shows that the system is effective and reliable. After using the system based on cloud computing, its low cost and scale effect promote the popularization of the system and expand information source greatly. More and more abundant information Gather together on the cloud data center, after fully sharing, integration and processing, deriving a lot of specific applications of flow information. Moreover, the flow integrating information system based on cloud computing is of a great significance for the design of other system.

\section{References}

[1] Huang Jianjun. Gas Flow Measurement Based on Temperature and Pressure Compensation. Control and Instruments in Chemical Industry, 40(1), pp. 1153-1159, 2013.

[2] Palash B P. Thermodynamics of a Classical Ideal Gas at Arbitrary Temperatures. International Journal of Modern Physics B, 17(7), pp. 1035-1041, 2003.

[3] Lee B S, Yan S X, Ma D. Aggregating IaaS Service. SRII Global Conference (SRII),29(2), pp. 335-338, 2011.

[4] Di Costanzo A, De Assuncao M D, Buyya R. Harnessing cloud technologies for a virtualized distributed computing infrastructure. IEEE Internet Computing, 13(5), pp. 213-221, 2009.

[5] Quan Zhengrui. Analysis of problem on measuring value correction formula of differential pressure type gas flow-metering. Experiments and Measurements in Fluid Mechanics,16(3), pp. 972-979, 2002.

[6] DeWitt DJ, Paulson E, Robinson E, Naughton J. An integrated computation and data management system. Proc of the VLDB Endowment, 9(2), pp. 399-411, 2008.

[7] Lu Mantao. Necessity of temperature and pressure correction in measurement of gas volume flow. Metallurgical Industry Automation, 32(4), pp. 33-42, 2009.

[8] Durst F, Al-Salaymeh A, Bradshaw P. The development of a pulsed-wired probe for measuring flow velocity with bandwidth. International Journal of Heat and Fluid Flow, 5(1), pp. 111-122, 2009.

[9] Armbrust M, Stoica I, Zaharia M. A view of cloud computing. Communications of the ACM, 6(2), pp. 877-889, 2010.

[10] Dean J, Ghemawat S. MapReduce: simplified data processing on large clusters. Communications of the ACM, 2(2), pp. 200-210, 2005. 